\title{
Fatores de risco e prevenção primária da hemorragia digestiva alta: revisão
}

\section{integrativa}

\author{
Risk factors and primary prevention of upper gastrointestinal bleeding: an integrative review \\ Factores de riesgo y prevención primaria de la hemorragia digestiva alta: una revisión integradora
}

Recebido: 13/12/2021 | Revisado: 18/12/2021 | Aceito: 16/02/2022 | Publicado: 25/02/2022

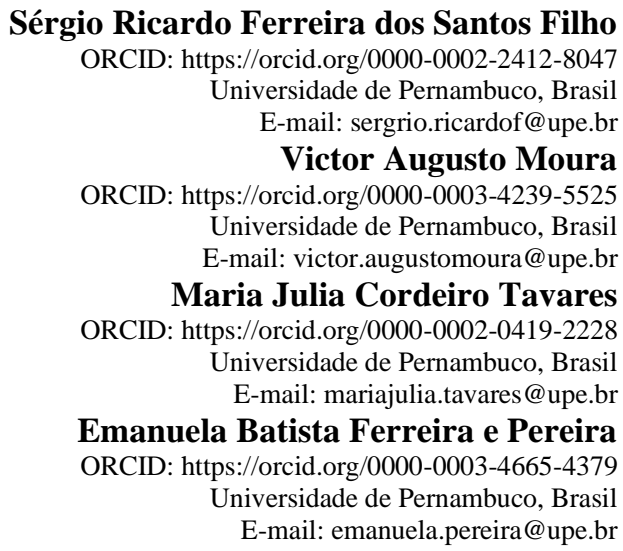

\begin{abstract}
Resumo
As hemorragias digestivas estão entre as urgências mais frequentes em serviços de saúde brasileiros, com necessidade de hospitalização numa elevada percentagem dos doentes, sendo as hemorragias digestivas altas mais prevalentes que as hemorragias digestivas baixas. Trata-se de uma revisão integrativa de literatura, realizada a partir da análise de artigos científicos com buscas baseadas em evidências e a utilização da estratégia do acrônimo PICO. O objetivo é avaliar, através de revisão integrativa, os principais fatores de risco para o desenvolvimento da hemorragia digestiva alta e a formas de profilaxia primária do sangramento. Após cruzamento dos descritores nas bases de dados e filtragem a partir dos critérios de inclusão e de exclusão, 6 estudos foram selecionados para comporem a amostra final. Os estudos reafirmam o que já é evidenciado na literatura, reafirmando como principais fatores de risco para o desenvolvimento da HDA: úlcera péptica, varizes esofágicas, cirrose hepática, uso de medicamentos agressores da mucosa gástrica como os anti-inflamatórios não esteróides, infecção por H. Pylori, sexo masculino, idade avançada, insuficiência renal e estilos de vida não saudáveis. Contudo, tais fatores de risco não são de domínio público no que tange a compreensão do que causam seus desenvolvimentos e quais riscos trazem à saúde. Faz-se necessário por parte dos profissionais em saúde maior dedicação à educação em saúde, principalmente no âmbito da baixa complexidade e atenção primária, promovendo a difusão de conhecimentos que proporcionem alicerce para o autocuidado.
\end{abstract}

Palavras-chave: Fatores de risco; Hemorragia gastrointestinal; Prevenção primária.

\begin{abstract}
Digestive hemorrhages are among the most frequent emergencies in Brazilian health services, requiring hospitalization in a high percentage of patients, with upper digestive hemorrhages being more prevalent than lower digestive hemorrhages. This is an integrative literature review, carried out from the analysis of scientific articles with evidencebased searches and the use of the acronym PICO strategy. The objective is to evaluate, through an integrative review, the main risk factors for the development of upper gastrointestinal bleeding and forms of primary bleeding prophylaxis. After crossing the descriptors in the databases and filtering based on the inclusion and exclusion criteria, 6 studies were selected to make up the final sample. The studies reaffirm what is already evidenced in the literature, reaffirming as the main risk factors for the development of $\mathrm{ADH}$ : peptic ulcer, esophageal varices, liver cirrhosis, use of drugs that damage the gastric mucosa such as nonsteroidal anti-inflammatory drugs, $\mathrm{H}$ infection Pylori, male gender, old age, kidney failure and unhealthy lifestyles. However, such risk factors are not in the public domain in terms of understanding what causes their development and what risks they bring to health. It is necessary on the part of health professionals greater dedication to health education, especially in the context of low complexity and primary care, promoting the dissemination of knowledge that provide a foundation for self-care.
\end{abstract}

Keywords: Risk factors; Gastrointestinal hemorrhage; Primary prevention. 


\begin{abstract}
Resumen
Las hemorragias digestivas se encuentran entre las emergencias más frecuentes en los servicios de salud brasileños, requiriendo hospitalización en un alto porcentaje de pacientes, siendo más prevalentes las hemorragias digestivas altas que las hemorragias digestivas inferiores. Se trata de una revisión integradora de la literatura, realizada a partir del análisis de artículos científicos con búsquedas basadas en evidencia y el uso del acrónimo estrategia PICO. El objetivo es evaluar, mediante una revisión integradora, los principales factores de riesgo para el desarrollo de hemorragia digestiva alta y formas de profilaxis de hemorragia primaria. Tras cruzar los descriptores en las bases de datos y filtrar en función de los criterios de inclusión y exclusión, se seleccionaron 6 estudios para conformar la muestra final. Los estudios reafirman lo ya evidenciado en la literatura, reafirmando como principales factores de riesgo para el desarrollo de HAD: úlcera péptica, varices esofágicas, cirrosis hepática, uso de fármacos que dañan la mucosa gástrica como antiinflamatorios no esteroideos, infección por H. Pylori, sexo masculino, vejez, insuficiencia renal y estilos de vida poco saludables. Sin embargo, tales factores de riesgo no son de dominio público en términos de comprender qué causa su desarrollo y qué riesgos traen a la salud. Es necesaria por parte de los profesionales de la salud una mayor dedicación a la educación para la salud, especialmente en el contexto de baja complejidad y atención primaria, promoviendo la difusión de conocimientos que sientan las bases del autocuidado.
\end{abstract}

Palabras clave: Factores de riesgo; Hemorragia gastrointestinal; Prevención primaria.

\title{
1. Introdução
}

As hemorragias digestivas estão entre as urgências mais frequentes em serviços de saúde brasileiros, com necessidade de hospitalização numa elevada percentagem dos doentes, sendo as hemorragias digestivas altas mais prevalentes que as hemorragias digestivas baixas e, apesar dos avanços na área diagnóstica e terapêutica, mantém-se com alta taxa de mortalidade (Melo, 2015).

A diferenciação entre hemorragia digestiva alta (HDA) e hemorragia digestiva baixa (HDB) é feita de acordo com a origem deste sangramento, tendo como parâmetro divisor o ligamento de Treitz. Caso a lesão que cause o sangramento seja proximal ao ligamento de Treitz (esôfago, estômago ou duodeno) caracteriza-se uma hemorragia digestiva alta, caso o motivo do sangramento seja distal ao ligamento de Treitz (intestino delgado, intestino grosso, reto e anus) caracteriza-se uma hemorragia digestiva baixa (Martins et al., 2019).

Assim, a hemorragia digestiva alta é definida como um sangramento derivado de lesões no trato gastrointestinal superior, podendo serem classificadas em HDA varicosa, quando o sangramento ocorre devido a rupturas de varizes presentes no TGI, provocadas por hipertensão portal decorrente de complicações hepáticas; e em HDA não-varicosa, que representam cerca de 80-90\% dos casos de HDA e tem como principal etiologia as úlceras pépticas ou vasculares, associadas ou não com infecção bacteriana (Martins et al., 2019).

O sangramento que ocorre no trato digestivo é um problema presenciado nos serviços de atendimentos emergenciais em todo o mundo, sendo evidenciado de forma frequente e comum. Contudo, a presença geral desta enfermidade não é muito conhecida, apesar de que a sua incidência anual varia entre 100-150 casos para cada 100.000 habitantes, que precisam ser hospitalizados de imediato por este motivo. Embora possa ser mais comum em homens, esta ocorrência pode-se presenciar em qualquer grupo demográfico, onde aumenta progressivamente com o avançar da idade (Araujo et al., 2016).

Aproximadamente 25\% dos atendimentos nos serviços de emergência brasileiras correspondem a ocorrências de HDA, aliada a uma mortalidade que tem se mantido elevada, com taxas entre $4 \%$ e $10 \%$ nos casos não varicosos e $18 \%$ e $30 \%$ nos casos de origem varicosa, e mesmo com os significativos avanços na área diagnóstica e terapêutica, o risco pode ser aumentado em até 70\% num episódio reincidente (Araujo et al., 2016).

As manifestações clínicas e laboratoriais da HDA dependem da velocidade e extensão da perda sanguínea, além da presença ou não de doenças concomitantes. Sinais sistêmicos raramente são apresentados numa perda inferior a $500 \mathrm{~mL}$ de sangue. Já em idosos e anêmicos, um pequeno sangramento já poderá produzir alterações hemodinâmicas (Wannmacher, 2007). Tais manifestações consistem, em quase totalidade de casos, em hematêmese (eliminação de sangue por êmese), podendo 
também apresentar melena (eliminação de sangue digerido junto às fezes) e em caráter de exceção poderá estar presente hematoquezia (eliminação de sangue vivo junto às fezes) (Martins et al., 2019).

Hemorragias digestivas são manifestações clínicas desafiantes mediante o seu tratamento. Faz-se necessário a determinação de um diagnóstico adequado e rápido, para precaver o desenvolvimento de complicações que possibilitam acarretar em óbito. Por isso, a comunicação entre os profissionais de saúde especializados e a correta utilização das tecnologias avançadas são de grande importância, bem como fatores determinantes no tratamento de pacientes, evitando assim, grandes cirurgias que podem ter o aumento dos custos e elevada mortalidade (Jiménez et al., 2019).

No contexto da recente pandemia de Sars-CoV-2, decretada oficialmente pela OMS em 11 de março de 2020, Mauro et al. (2020) diz que o risco e incidência da HDA em pacientes com Covid-19 é desconhecido, mas aqueles que desenvolvem o quadro grave da doença estão mais suscetíveis ao desenvolvimento da úlcera péptica de stress, já que necessitam de cuidados intra-hospitalares avançados em unidades de terapia intensiva (UTIs), onde essa comorbidade é mais frequente, aumentando muito as chances de ocorrência de episódios de sangramento no trato gastrointestinal (TGI).

Em serviços de emergências no Brasil e no mundo, rotineiramente, episódios de HDA são controlados por meio da endoscopia digestiva, com base em diretrizes e guidelines nacionais e internacionais, no entanto, os benefícios da endoscopia digestiva para identificação precoce de úlceras pépticas de stress entram em conflito com possíveis complicações que podem surgir durante o exame, já que são pacientes que têm um risco aumentado para uma piora do quadro respiratório durante a endoscopia, podendo agravar-se ao ponto da necessidade de suporte ventilatório avançado e transferência para um leito de UTI, que seria um problema crítico durante o pico do surto de Covid-19, onde as demandas por leitos de UTI, que já mostravam-se altas antes mesmo do cenário pandêmico, elevaram-se exponencialmente nesse período (Mauro et al., 2021).

Constitui-se de elevada necessidade também, avaliar a efetivação das declarações baseadas em evidências, para entender quais as dificuldades são tidas a este respeito, identificar pontos suscetíveis a melhorias e planejar a implementação das intervenções mais apropriadas, com a intenção final de melhorar a qualidade da atenção à saúde dos indivíduos que possam ser acometidos por este agravo clínico (Benites-Goñi et al., 2020).

Atualmente, a maior parcela dos usuários que procuram um serviço de saúde de urgência correspondem a habitantes de áreas urbanas-periféricas e de baixo estrato socioeconômico (Condo, 2018), onde fatores de risco agressores da mucosa gástrica e do sistema digestivo estão presentes, como a infeção por H. pylori e o uso recorrente de AINEs, que juntos, elevam de 3 a 5 vezes o risco de acometimento por hemorragia digestiva alta (Martins et al., 2019), além do alcoolismo e de hábitos de vida e alimentares não saudáveis.

Cerca de 73,8\% dos pacientes que tiveram HDA, apresentavam estilos de vida com hábitos agressores da mucosa gástrica e do sistema digestivo, e a problemática persistente em relação à morbimortalidade em pacientes acometidos pela HDA, torna necessário que esforços sejam gerados com a finalidade de se garantir políticas de prevenção e promoção da saúde. (Araujo et al., 2016).

Este estudo justifica-se pela importância de identificar e mapear os fatores de risco associados a hemorragia digestiva alta, a fim de reduzir sua incidência na população, com o desenvolvimento de ações no nível de complexidade primário, já que a prevenção do primeiro episódio de HDA é crucial para reduzir a morbimortalidade e os custos no sistema de saúde.

O objetivo deste estudo é avaliar, através de revisão integrativa, os principais fatores de risco para o desenvolvimento da hemorragia digestiva alta e a formas de profilaxia primária do sangramento. 


\section{Metodologia}

Trata-se de uma revisão integrativa de literatura, realizada a partir da análise de artigos científicos com buscas baseadas em evidências e a utilização da estratégia do acrônimo PICO (população, intervenção, comparação e outcomes) na elaboração do problema de pesquisa.

Esse tipo de estudo caracteriza-se pela agregação das pesquisas obtidas de estudos, sobre uma temática investigada, realizados em metodologias diversas, promovendo ao revisor produzir uma análise e sintetização dos dados primários de forma rigorosa e sistemática (Soares et al., 2014).

O acrônimo PICO consiste na representação de quatro elementos fundamentais e eficientes para a elaboração de um problema de pesquisa. São eles: Paciente, Intervenção, Comparação e "Outcomes" (resultados). Essa estratégia permite ao pesquisador construir questões de estudo de propriedades diversas, originadas da clínica, do gerenciamento de recursos humanos e materiais, da averiguação de instrumentos para a avaliação de sintomas, dentre outros (Santos et al., 2017).

Na construção de uma revisão integrativa de literatura, são desenvolvidos os seguintes critérios: elaboração do problema de pesquisa; busca ou amostragem na literatura; coleta e análise dos dados; discussão dos resultados, por fim, a explanação dos resultados da revisão (Souza et al., 2010).

O presente estudo busca investigar na literatura as principais causas que levam a manifestação HDA, conduzidas pelo seguinte problema de pesquisa: Quais os principais fatores de risco para o desenvolvimento da hemorragia digestiva alta e quais as formas de profilaxia primária descritos na literatura?

O estudo foi realizado através das plataformas digitais de pesquisas Scientific Electronic Library Online (SciELO) e pela Biblioteca virtual em saúde (BVS) com as consecutivas bases de dados: Literatura Latino-americana e do Caribe em Ciências da Saúde (LILACS) e National Library of Medicine (PubMed). A amostragem foi constituída por artigos científicos publicados nessas bases de dados e bibliotecas virtuais conforme citadas.

Neste estudo, foram incluídos os artigos originais, disponíveis na íntegra e de acesso gratuito, publicados em português, inglês e espanhol, na qual o período de publicação seja entre 2016 a 2021, cujo objeto de estudo evidencie os fatores de risco e prevenção primária da HDA.

Foram considerados parâmetros de exclusão artigos duplicados entre as bases de dados, em formato de resenhas, relatos de caso/experiências, teses, anais de congressos e também aqueles que são em formatos de resumos.

Os descritores em português utilizados para a estratégia de busca dos artigos que constituíram os resultados deste estudo, de acordo com o sítio eletrônico Descritores em Ciências da Saúde (Decs), são os seguintes: Fatores de Risco, Hemorragia Gastrointestinal e Prevenção Primária, e as respectivas traduções dos seus correspondentes em inglês (Risk Factors; Gastrointestinal Hemorrhage and Primary Prevention) e em espanhol (Factores de Riesgo; Hemorragia Gastrointestinal y Prevención Primaria) foram obtidas através do Medical Subject Headings (MeSH).

Diante disso, a expressão de busca foi aplicada pelo operador booleano AND entre os termos, a partir dos cruzamentos dos descritores, para o processo de identificação dos artigos nas bases de dados e bibliotecas. A seleção dos artigos foi realizada seguindo as recomendações do fluxograma PRISMA, do inglês Transparent Reporting of Systematic Reviews and MetaAnalysis, que tem o objetivo de colaborar com os pesquisadores a aperfeiçoarem a qualidade de suas revisões de literatura (Galvão et al., 2015).

A classificação dos estudos e de suas evidências foi realizada através do Sistema GRADE, que trata-se de um método criado por uma equipe colaborativa de pesquisadores, a fim de criar um sistema universal, transparente e sensível para categorizar a qualidade das evidências e a força das recomendações.

Os níveis de evidência de acordo com o GRADE são qualificados em quatro níveis: alto - classificados pela forte confiança de que o efeito encontra-se próximo do estimado, tendo como exemplo ensaios clínicos bem delineados; moderado - 
na qual a confiança no efeito esperado é moderada, embasados em ensaios clínicos leves, onde futuros estudos podem mudar a confiança das evidências; baixo - a confiança no efeito é limitada, onde estudos posteriores possivelmente terão impacto importante na confiança dos achados, comumente representados em ensaios clínicos com limitações moderadas e estudos observacionais comparativos de coorte e controle e; muito baixo - onde a confiança é bastante limitada, com um importante nível de incerteza nos achados do estudo, qualquer suposição é de caráter de incerteza. Tais níveis retratam a convicção que temos na estimativa dos efeitos exibidos (Brasil, 2014).

A interpretação das evidências obtidas foi complementada através de uma contraposição das mesmas à luz da literatura especializada, diretrizes nacionais e guidelines de associações internacionais visando a identificação e descrição dos principais fatores de risco para o desenvolvimento da hemorragia digestiva alta, seguido de um apontamento das principais condutas de profilaxia, a nível de atenção primária à saúde, da hemorragia digestiva alta.

Este estudo não foi submetido à autorização do Comitê de Ética e Pesquisa, pois trata-se de uma revisão integrativa de literatura, portanto não há pesquisa envolvendo seres humanos em nenhuma fase do seu desenvolvimento e, consequentemente, dispensa a obrigatoriedade das recomendações previstas pela Resolução 466/2012, do Conselho Nacional de Saúde.

\section{Resultados}

A coleta de dados ocorreu durante os meses de junho, julho e agosto de 2021, com filtragem dos artigos nas bases de dados de acordo com os critérios estabelecidos e com auxílio da ferramenta Rayyan.

A partir do cruzamento dos descritores nas bases de dados, conforme descrito na metodologia do estudo e exposto a seguir no Quadro 1, foram encontrados 348 artigos, sendo 302 deles obtidos na PubMed, 36 na LILACS e os 10 últimos na SciELO.

Quadro 1. Processo de identificação dos artigos nas bases de dados. Recife, 2021.

\begin{tabular}{|c|c|c|c|c|}
\hline & $\begin{array}{c}\text { Fatores de risco AND } \\
\text { Hemorragia } \\
\text { gastrointestinal }\end{array}$ & $\begin{array}{c}\text { Hemorragia } \\
\text { gastrointestinal AND } \\
\text { Prevenção primária }\end{array}$ & $\begin{array}{c}\text { Fatores de risco AND } \\
\text { Hemorragia gastrointestinal AND } \\
\text { Prevenção primária }\end{array}$ & TOTAL \\
\hline PubMed & 283 & 15 & 4 & 302 \\
\hline LILACS & 34 & 1 & 1 & 36 \\
\hline SciELO & 7 & 2 & 1 & 348 \\
\hline TOTAL & 324 & 18 & 6 & 348 \\
\hline
\end{tabular}

Fonte: Dados da pesquisa (2021).

Dos artigos encontrados, 32 deles foram excluídos da amostra por serem duplicados ou não possuírem textos completos disponíveis gratuitamente, restando 316 artigos pré-selecionados para análise.

Após leitura flutuante dos 316 artigos, 262 deles foram excluídos por não estarem de acordo com a temática, com o objetivo ou não responderem a pergunta norteadora, restando 54 deles que foram considerados potencialmente elegíveis para comporem a amostra final. No entanto, após análise mais aprofundada desses 54 trabalhos, 47 deles foram excluídos, onde: 8 deles não eram artigos, 3 deles eram teses e 19 deles não abordavam o objetivo do estudo, não respondiam à pergunta norteadora ou foram enquadrados em algum outro critério de exclusão que foram estabelecidos, sendo tal etapa ilustrada na Figura 1: 
Figura 1. Fluxograma prisma da seleção dos artigos. Recife, 2021.

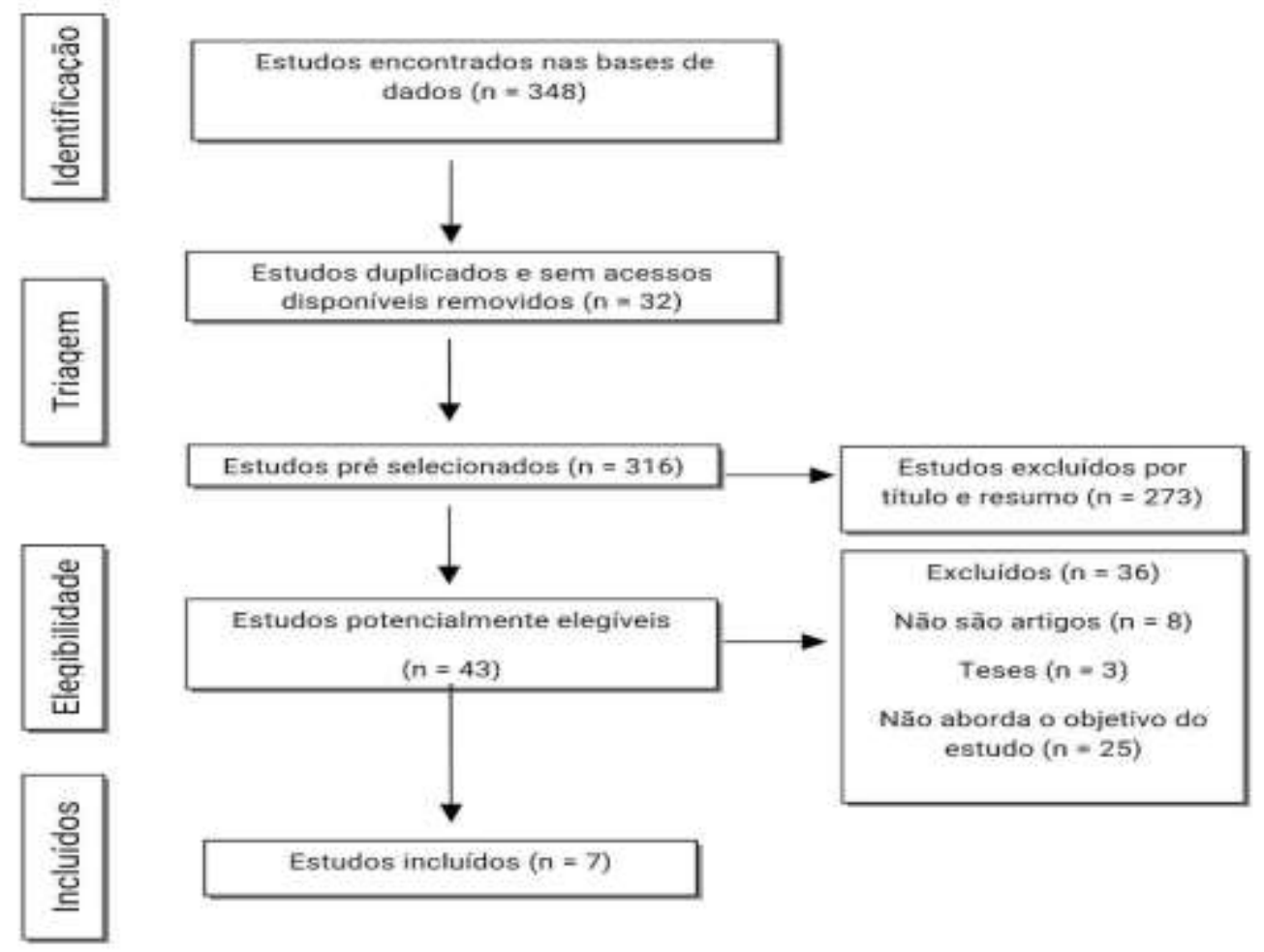

Fonte: Protocolo PRISMA.

Ao final, 7 estudos restaram e foram selecionados para comporem a amostra final e servirem de base para a realização da revisão, por atenderem aos critérios de inclusão, abordarem a temática e estarem em consonância com a pergunta norteadora.

O resultado da análise dos dados é avaliar os artigos que compuseram a amostra final, expondo as principais características dos 6 estudos incluídos na amostra final numa tabela com as seguintes variáveis: autor, título do artigo, base de dados, ano de publicação, tipo de estudo, número de amostra, os principais fatores de risco e formas de prevenção primária relatadas nos artigos incluídos no estudo (Quadro 2), na qual foi observada uma predominância de artigos na modalidade observacional. 
Quadro 2. Caracterização dos estudos selecionados quanto a autor, ano, país, tipo de estudo, amostra, tempo de execução e local do estudo. Recife, 2021.

\begin{tabular}{|c|c|c|c|c|c|c|}
\hline Estudo & Autor/Ano & País & Tipo de estudo & Amostra & Tempo de execução & Local do estudo \\
\hline E1 & $\begin{array}{l}\text { Kim et al. } \\
2016\end{array}$ & $\begin{array}{l}\text { Coreia do } \\
\text { Sul }\end{array}$ & $\begin{array}{c}\text { Estudo } \\
\text { observacional }\end{array}$ & 801.926 & 132 meses & $\begin{array}{l}\text { Ambulatório e } \\
\text { unidades de } \\
\text { internação }\end{array}$ \\
\hline E2 & $\begin{array}{l}\text { Kiringa et al. } \\
2020\end{array}$ & Uganda & $\begin{array}{l}\text { Estudo } \\
\text { transversal }\end{array}$ & 150 & 8 meses & $\begin{array}{l}\text { Unidades de } \\
\text { internação }\end{array}$ \\
\hline E3 & $\begin{array}{c}\text { Moledina; Komba. } \\
2017\end{array}$ & Tanzânia & Estudo de coorte & 170 & 7 meses & $\begin{array}{l}\text { Unidades de } \\
\text { internação }\end{array}$ \\
\hline E4 & $\begin{array}{l}\text { Lee et al } \\
2016 \text {. }\end{array}$ & $\begin{array}{l}\text { Coreia do } \\
\text { Sul }\end{array}$ & $\begin{array}{c}\text { Estudo } \\
\text { observacional }\end{array}$ & 336 & 32 meses & $\begin{array}{l}\text { Unidades de } \\
\text { internação }\end{array}$ \\
\hline E5 & $\begin{array}{l}\text { Pinto et al. } \\
2020\end{array}$ & Chile & $\begin{array}{c}\text { Estudo } \\
\text { observacional }\end{array}$ & 249 & 36 meses & $\begin{array}{l}\text { Unidades de } \\
\text { internação }\end{array}$ \\
\hline E6 & $\begin{array}{l}\text { Tang; Xiao. } \\
2020\end{array}$ & China & $\begin{array}{l}\text { Estudo caso- } \\
\text { controle }\end{array}$ & 388 & 33 meses & Emergência \\
\hline E7 & $\begin{array}{l}\text { Kim et al. } \\
2018\end{array}$ & Coreia & Estudo de coorte & 904 & 10 meses & $\begin{array}{l}\text { Unidades de } \\
\text { internação }\end{array}$ \\
\hline
\end{tabular}

Fonte: Dados da pesquisa (2021).

Estes 7 estudos também estão caracterizados de acordo com os conhecimentos produzidos sobre o tema, seus objetivos, seus níveis de evidência de acordo com a metodologia GRADE, os fatores de risco evidenciados para HDA e breves resumos de seus resultados e conclusão no Quadro 3:

Quadro 3. Caracterização dos estudos selecionados quanto ao objetivo, nível de evidência, fatores de risco e resultados/conclusão. Recife, 2021.

\begin{tabular}{|c|c|c|c|c|}
\hline Estudo & Objetivo & $\begin{array}{l}\text { Nível de } \\
\text { evidência } \\
\text { (GRADE) }\end{array}$ & Fatores de Risco & $\begin{array}{l}\text { Resultados } \\
\text { Conclusão }\end{array}$ \\
\hline E1 & $\begin{array}{l}\text { Avaliar a prevalência de } \\
\text { possíveis fatores de risco de } \\
\text { hemorragia digestiva alta } \\
\text { (UGIB) e sua tendência } \\
\text { específica de faixa etária na } \\
\text { população em geral e pacientes } \\
\text { com osteoartrite. }\end{array}$ & $\begin{array}{c}\text { Moderado } \\
\mathrm{p}=0,20\end{array}$ & $\begin{array}{l}\text { Idade, doença hepática, } \\
\text { tabagismo, alcoolismo, úlcera } \\
\text { péptica, uso de medicamentos } \\
\text { concomitantes, uso de anti- } \\
\text { inflamatórios não esteroides } \\
\text { (AINEs), infecção por } \\
\text { Helicobacter pylori e } \\
\text { osteoartrite. }\end{array}$ & $\begin{array}{l}\text { A prevalência de fatores de risco múltiplos } \\
\text { individuais e simultâneos tornou-se maior } \\
\text { com o aumento da idade, onde cada } \\
\text { condição comórbida, hábitos pessoais e } \\
\text { uso de medicamentos concomitantes foi } \\
\text { maior em pacientes com osteoartrite. } \\
35,02 \% \text { da população geral e } 68,50 \% \text { dos } \\
\text { pacientes com osteoartrite tinham pelo } \\
\text { menos um ou mais fatores de risco de } \\
\text { UGIB. }\end{array}$ \\
\hline
\end{tabular}




\begin{tabular}{|c|c|c|c|c|}
\hline E2 & $\begin{array}{l}\text { "Determinar prevalência dos a } \\
\text { sintomas e da taxa de letalidade } \\
\text { de hemorragia digestiva alta } \\
\text { entre os pacientes do serviço de } \\
\text { gastroenterologia do Hospital } \\
\text { Mulago em Kampala, Uganda e } \\
\text { descrever os fatores de risco } \\
\text { clínicos e laboratoriais } \\
\text { associados à sobrevida desses } \\
\text { pacientes." }\end{array}$ & $\begin{array}{c}\text { Baixo } \\
\mathrm{p}<0,05\end{array}$ & $\begin{array}{l}\text { Sexo masculino, varizes } \\
\text { esofágicas, hipertensão portal, } \\
\text { úlcera peptica, cirrose } \\
\text { hepática, gastrite, uremia, } \\
\text { malignidade, infecção por } \\
\text { Helicobacter pylori e } \\
\text { alcoolismo. }\end{array}$ & $\begin{array}{l}\text { A prevalência de sintomas e a taxa de } \\
\text { letalidade de hemorragia digestiva alta } \\
\text { entre os pacientes internados foram } \\
\text { maiores do que em países desenvolvidos e } \\
\text { semelhantes a outras configurações de } \\
\text { recursos limitados. A taxa geral de } \\
\text { letalidade foi de } 16,7 \% \text {. A análise de } \\
\text { sobrevida indica que o sexo masculino, } \\
\text { uremia e malignidade estão associados à } \\
\text { redução da mesma. }\end{array}$ \\
\hline E3 & $\begin{array}{c}\text { Identificar as causas da } \\
\text { hemorragia digestiva alta e os } \\
\text { fatores que aumentam o risco de } \\
\text { mortalidade nesses pacientes. }\end{array}$ & $\begin{array}{c}\text { Alto } \\
\mathrm{p}=0,05\end{array}$ & $\begin{array}{l}\text { Varizes esofágicas, doença } \\
\text { hepática crônica, úlcera } \\
\text { péptica, gastrite, sexo } \\
\text { masculino, contagem elevada } \\
\text { de leucócitos, alanina } \\
\text { aminotransferase sérica } \\
\text { elevada e bilirrubina total } \\
\text { sérica elevada. }\end{array}$ & $\begin{array}{l}\text { As varizes esofágicas foram a causa mais } \\
\text { comum de UGIB. Um terço dos pacientes } \\
\text { admitidos com sangramento } \\
\text { gastrointestinal alto morreu dentro de } 60 \\
\text { dias da admissão, o que significa uma } \\
\text { carga elevada. Esses achados podem ser } \\
\text { usados para estratificar o risco de } \\
\text { pacientes que podem se beneficiar de um } \\
\text { tratamento precoce e mais agressivo." }\end{array}$ \\
\hline E4 & $\begin{array}{l}\text { Avaliar os fatores preditivos de } \\
\text { mortalidade no período de } 30 \\
\text { dias em pacientes com } \\
\text { hemorragia digestiva alta. }\end{array}$ & $\begin{array}{c}\text { Baixo } \\
\mathrm{p}<0,05\end{array}$ & $\begin{array}{c}\text { Diabetes melittus, } \\
\text { malignidade metastática, sexo } \\
\text { masculino, idade } \geq 65 \text { anos e } \\
\text { instabilidade hemodinâmica } \\
\text { durante hospitalização. }\end{array}$ & $\begin{array}{l}\text { O risco de mortalidade em } 30 \text { dias em } \\
\text { pacientes com hemorragia digestiva alta } \\
\text { foi significativamente maior em pacientes } \\
\text { com idade avançada, comorbidade de DM } \\
\text { ou malignidade metastática e instabilidade } \\
\text { hemodinâmica durante a hospitalização. } \\
\text { Esses achados sugerem que o manejo } \\
\text { agressivo e o monitoramento cuidadoso de } \\
\text { acordo com diretrizes específicas devem } \\
\text { ser fornecidos para pacientes de alto risco. } \\
\text { Esses resultados ajudarão a orientar o } \\
\text { manejo de pacientes com essa condição. }\end{array}$ \\
\hline E5 & $\begin{array}{l}\text { Determinar a mortalidade } \\
\text { hospitalar e os fatores de risco } \\
\text { associados em pacientes } \\
\text { hospitalizados com hemorragia } \\
\text { digestiva alta e comparar as } \\
\text { características clínicas e } \\
\text { desfechos de pacientes com } \\
\text { HDA varicosas e não varicosas. }\end{array}$ & $\begin{array}{c}\text { Moderado } \\
\mathrm{p}<0,05\end{array}$ & $\begin{array}{c}\text { Idade } \geq 60 \text { anos, sexo } \\
\text { masculino, úlcera péptica, } \\
\text { síndrome de Mallory-Weiss, } \\
\text { lesões de Dieulafoy, } \\
\text { creatinina elevada, transfusão } \\
\text { de hemácias e sangramento } \\
\text { de varizes esofágicas. }\end{array}$ & $\begin{array}{l}\text { A mortalidade hospitalar de hemorragia } \\
\text { digestiva alta permanece elevada, } \\
\text { especialmente nas varicosas. transfusão de } \\
\text { hemácias (odds ratio (OR): } 18,7, \mathrm{p}<0,01 \text { ), } \\
\text { creatinina elevada na admissão (OR: } 3,30 \text {, } \\
\mathrm{p}=0,03 \text { ) e sangramento de varizes (OR: } \\
3,23, \mathrm{p}=0,02 \text { ) são os principais fatores de } \\
\text { risco para a mortalidade. }\end{array}$ \\
\hline E6 & $\begin{array}{l}\text { Analisar os fatores de risco para } \\
\text { sangramento gastrointestinal } \\
\text { alto (UGIB) em pacientes com } \\
\text { infarto do miocárdio com } \\
\text { supradesnivelamento do } \\
\text { segmento ST (IAMCSST) } \\
\text { durante terapia antiplaquetária } \\
\text { dupla (DAPT) após intervenção } \\
\text { coronária percutânea (ICP). }\end{array}$ & $\begin{array}{c}\text { Moderado } \\
\mathrm{p}<0,05\end{array}$ & $\begin{array}{c}\text { Idade avançada, tabagismo, } \\
\text { infecção por Helicobacter } \\
\text { pylori, história de doenças do } \\
\text { trato digestivo e insuficiência } \\
\text { renal. }\end{array}$ & $\begin{array}{c}\text { Em comparação com o grupo controle, o } \\
\text { grupo com sangramento gastrointestinal } \\
\text { alto teve internação hospitalar } \\
\text { significativamente mais longa, e maior } \\
\text { proporção de descontinuação de } \\
\text { medicamentos antitrombóticos e taxa de } \\
\text { mortalidade }(\mathrm{P}<0,05) \text {. O uso adequado de } \\
\text { PPI depois disso pode reduzir a taxa de } \\
\text { incidência de hemorragia digestiva alta. }\end{array}$ \\
\hline
\end{tabular}




\begin{tabular}{|c|c|c|c|c|}
\hline E7 & $\begin{array}{c}\text { Fatores associados a } \\
\text { ressangramento em pacientes } \\
\text { com sangramento de úlcera } \\
\text { péptica: análise do estudo } \\
\text { coreano de sangramento de } \\
\text { úlcera péptica. }\end{array}$ & $\begin{array}{c}\text { Moderado } \\
\mathrm{p}<0,5\end{array}$ & $\begin{array}{c}\text { A presença de comorbidades, uso de } \\
\text { múltiplos medicamentos, níveis de } \\
\text { Os fatores de risco para } \\
\text { ressangramento foram a } \\
\text { presença de comorbidades, } \\
\text { uso de múltiplos } \\
\text { medicamentos, níveis de } \\
\text { albumina e hematêmese / } \\
\text { hematoquezia como } \\
\text { hematêmese / hematoquezia podem ser } \\
\text { apresentações iniciais. }\end{array}$ & $\begin{array}{c}\text { indicadores de ressangramento nesses } \\
\text { pacientes. O amplo uso de inibidores da } \\
\text { bomba de prótons e intervenções } \\
\text { endoscópicas imediatas podem explicar a } \\
\text { baixa incidência de ressangramento e as } \\
\text { baixas taxas de mortalidade na Coréia. }\end{array}$ \\
\hline
\end{tabular}

Fonte: Dados da pesquisa (2021).

\section{Discussão}

Apesar dos avanços da ciência na área da saúde em relação a diagnósticos e terapêuticas e taxa de mortalidade ter diminuído consideravelmente nos últimos anos, a hemorragia digestiva alta ainda se apresenta como uma enfermidade de alto risco, com elevada morbimortalidade e os fatores que levam a esta condição são bastante evidenciados na literatura. Os casos de óbito intra-hospitalar podem variar entre 4,4\% e 13\% nas hemorragias digestivas não varicosas e varicosas, respectivamente. Sendo as hemorragias digestivas altas não varicosas mais frequentes que as de origem varicosa (Pinto et al., 2020).

Os estudos reafirmam o que já é evidenciado na literatura como principais fatores de risco para o desenvolvimento da HDA: úlcera péptica, esofagite erosiva, varizes esofágicas, cirrose hepática, uso de medicamentos agressores da mucosa gástrica como os anti-inflamatórios não esteróides (AINEs), infecção por Helicobacter Pylori, sexo masculino, idade avançada, insuficiência renal e estilos de vida não saudáveis, com destaque para alcoolismo e tabagismo. A lesão de Dieulafoy é mencionada como um importante, mas não comum, fator de risco para a HDA.

A úlcera péptica (DUP) é um distúrbio frequentemente relacionado a sua capacidade de interferir de forma desfavorável na qualidade de vida e no rendimento das atividades diárias do portador da mesma. A prevalência desta enfermidade apresentase comumente nos casos de sangramento gastrointestinal alto e segundo Kiringa et al. (2020) e Pinto et al. (2020) em seus estudos, a DUP classifica-se como a principal causa de HDA não varicosa, com $26 \%$ e $44 \%$ dos achados endoscópicos, respectivamente. Acredita-se que esta frequência se dá pela alta predominância de infecção por H. pylori na mucosa gástrica, causador determinante da DUP, somado à elevada ingestão de anti-inflamatórios não esteróides e arterite, uma inflamação das paredes arteriais, causadas sobretudo pela exposição a secreção cloridropéptica. As DUP são descritas de acordo com a classificação de Forrest, podendo variar entre "III- base limpa" e "Ia - Sangramento ativo em jato" (Giordiano-Nappi \& Filho, 2008; Soares et al, 2018).

A infecção por Helicobacter pylori, uma bactéria gram negativa que coloniza a mucosa gástrica causando gastrite atrófica está fortemente associada à patogênese das úlceras gástricas e duodenais, carcinomas gástricos e linfomas. Esta infecção está relacionada com más práticas sanitárias, como a não higienização das mãos e alimentos pré-refeição. Já os AINEs são considerados um fator muito importante na cronificação das úlceras, além de apresentarem efeito tóxico na mucosa gastroduodenal, que têm como função lubrificar e proteger o epitélio intestinal. O tratamento com AINEs também é o causador do desenvolvimento de petéquias, equimoses, erosões, úlceras e, eventualmente, perfuração ou estenose. Cerca de 10 a $30 \%$ dos pacientes desenvolvem úlceras pépticas em 6 meses com tratamento contínuo (Condo, 2018).

Dentre os medicamentos agressores da mucosa gástrica, o uso de aspirina e AINEs para o tratamento de dores crônicas e agudas advindas de um processo inflamatório vem aumentando nos últimos anos, consequentemente elevando os riscos de HDA (Kim et al., 2016; Lee et al., 2016). Segundo Kim et al. (2016), pacientes com osteoartrite apresentam-se como grandes consumidores desses medicamentos e quando se leva em comparação com a população geral, a frequência desse uso é muito 
maior. Inclusive neste estudo, apesar de a aspirina ser o medicamento mais usado na população geral estudada, os AINEs tiveram maior predomínio nos indivíduos com esses problemas articulares. O risco de complicações gastrointestinais aumenta cerca de 5 vezes quando comparado à população que não faz a utilização desses fármacos, sendo imprescindível que os profissionais de saúde preescrevam esses medicamentos de acordo com a necessidade de cada paciente, avaliando o custo-benefício de forma individual, além de incluírem indicações de proteção gástrica como inibidores de bombas de prótons (Couto et al, 2010).

Aliado a esses fatores, o consumo excessivo de alimentos que fomentam o aumento do $\mathrm{pH}$ gástrico e/ou ardência e queimação como frituras, alimentos e bebidas cítricas, bebidas gaseificadas, chás, embutidos, biscoitos recheados, alimentos ricos em gorduras (queijos, carnes vermelhas) e de condimentação excessiva criam um ambiente propício para surgimento das úlceras pépticas (Araujo et al., 2016). Além disso, o álcool e a nicotina podem também causar desequilíbrio e prejudicar a mucosa gastrintestinal, inibindo a secreção de muco e bicarbonato, e aumentando a secreção ácida. Sendo necessária uma reeducação alimentar, onde o paciente possa promover a recuperação do tecido através de um cuidado nutricional, a fim de evitar uma hipersecreção cloridropéptica (Vomero, 2014).

Tang e Xiao (2020), mostram que drogas antiplaquetárias necessárias para o tratamento de pacientes cardiopatas coronarianos, como aspirina e clopidogrel aumentam o risco de sangramentos. A aspirina por inibir a produção de tromboxano, bloqueando a ciclooxigenase-1, inibindo a agregação plaquetária e afetando a mucosa gástrica durante a absorção do fármaco, deixando a mucosa gástrica desprotegida contra o próprio ácido clorídrico, favorecendo o surgimento de úlceras gástricas. Já o clopidogrel atua inibindo o receptor ADP que bloqueia o receptor P2Y12 após ativação pelo fígado, apesar de seu mecanismo de ação não ser muito bem elucidado, contribui para o sangramento por combater a agregação plaquetária.

O sangramento por varizes esofágicas, que tem como principal etiologia a hipertensão portal, ocorrendo uma obstrução do fluxo sanguíneo para o fígado e elevando a pressão da veia porta, comumente em pacientes cirróticos, é um fator de risco que apresenta alta taxa de mortalidade e maior gravidade na admissão intra-hospitalar, comparados com aqueles que possuem etiologia não varicosa (Pinto et al., 2020). No estudo de Moledina e Komba (2017), varizes esofágicas foi a causa mais comum de hemorragia digestiva alta entre os pacientes submetidos à endoscopia, identificado em 57\% dos mesmos. Entre as pessoas que realizaram endoscopia no estudo de Kiringa et al. (2020), o sangramento de varizes do esôfago também se apresentou como o principal fator de risco $(42,2 \%)$.

A cirrose é uma lesão hepática crônica onde as células do fígado são destruídas e esses danos não podem ser revertidos, o que afeta diretamente na funcionalidade do órgão. Pacientes que possuem essa comorbidade, diante de um quadro de DUP, possuem um risco de 2,62 vezes maior de desenvolver um sangramento em ambiente hospitalar (Kim et al., 2017).

Referente ao sexo, estudos afirmam que pessoas do sexo masculino são mais propensas a desenvolver hemorragia digestiva alta (Moledina \& Komba, 2017; Kiringa et al., 2020; Pinto et al., 2020). Corroborando a afirmativa anterior, Moledina e Komba (2017) em seu estudo, mostram que dentre os pacientes incluídos, os homens representaram a maioria com cerca de $71,2 \%$, contudo as causas dessa propensão não são bem definidas e ainda é um tema discutível na literatura.

No entanto, no processo de envelhecimento, o enfraquecimento das funções orgânicas e fisiológicas são evidentes, portanto, a taxa de episódios de hemorragia digestiva alta aumenta consideravelmente em pessoas com idade avançada. A função da mucosa gástrica diminui com o passar dos anos e o fornecimento de sangue é insuficiente para reparação da mesma, deixando o organismo propenso à vulnerabilidades (Tang \& Xiao, 2020). Aliado a esses fatores, idosos são mais susceptíveis a desenvolverem comorbidades diversas, que podem estar relacionadas a um maior risco de ressangramento, onde o uso concomitante de medicamentos agressores do sistema gastrointestinal é bastante comum. (Lee et al., 2016).

Os estudos de Lee et al. (2016); Moledina e Komba (2017) e Pinto et al. (2020) mencionam a escala de Rockall, que consiste num sistema de pontuação de risco desenvolvida em 1996 e tem como objetivo fazer uma estratificação de risco de recidiva e morte após episódio de HDA, incorporando aspectos endoscópicos e não endoscópicos, pontuando com base na idade 
do paciente, a pressão arterial sistólica, a frequência cardíaca para avaliar estado hemodinâmico, a presença de outras comorbidades e achados advindos da endoscopia digestiva, como hemorragias ativas e coágulos aderentes (Giordiano-Nappi; Filho, 2008). A pontuação da escala vai de 0 , como sendo a pontuação mínima, até a pontuação máxima de 11 . A pontuação obtida após aplicação da escala e somatória dos pontos pode se enquadrar em baixo risco, quando o resultado fica entre 0 e 2 pontos; médio risco quando o resultado fica entre 3 e 4 pontos; e alto risco quando a pontuação fica acima de 5 pontos, indicando uma estimativa de taxa de mortalidade de $40 \%$.

O escore de Glasgow-Blatchford (EGB), também mencionado nos estudos de Lee et al. (2016); Moledina e Komba (2017) e Pinto et al. (2020), utiliza de aspectos não endoscópicos para elaboração de uma pontuação, servindo como uma ferramenta de triagem para avaliar a probabilidade de um episódio de HDA necessitar de intervenção médica, como transfusão de sangue ou intervenção endoscópica. O escore pontua com base na uréia, hemoglobina, pressão arterial sistólica, frequência de pulso, presença de melena, síncope, hepatopatia e insuficiência cardíaca. Um somatório de pontuação igual a 0 corresponde a um paciente de baixo risco, que poderá receber alta precoce, já um somatório de pontuação igual a 1 ou superior corresponde a um paciente de alto risco de intervenção ou morte.

Nesse contexto, a endoscopia, que é um exame indicado para quadros de gastrite, úlcera péptica, esofagite, hérnia de hiato, cânceres do trato gastrointestinal e principalmente varizes esofágicas e HDAs, tem tido um crescente incremento em sua demanda, sendo também utilizado como forma de deteç̧ão e identificação precoce de tais complicações, podendo ser recomendado quando há a presença de sinais de dor abdominal, disfagia, perda de peso, odinofagia, anorexia, vômitos persistentes de causa desconhecida, ingestão aguda de cáusticos e biópsias (Souza, 2017).

O tratamento endoscópico é uma alternativa que traz bons resultados com baixas taxas de complicações. A Ligadura Elástica de Varizes Esofágicas (LEVE) é um procedimento que tem como objetivo a retração do cordão varicoso, impedindo o fluxo sanguíneo e, consequentemente, atuando como uma prevenção primária da hemorragia varicosa esofágica. Sua indicação é feita tanto para pacientes em quadros agudos quanto para aqueles que desejam prevenir uma recidiva (ElRahim, 2018).

Em Unidades de Terapia Intensiva (UTI), recomenda-se a profilaxia com inibidores de bombas de prótons no tratamento de úlceras por estresse, após ensaios clínicos mostrarem que há redução na incidência de sangramentos gastrointestinais. Esses pacientes são mais propícios a lesionarem a mucosa do TGI. Estas lesões crescem progressivamente com o passar dos dias e podem acarretar nesses sangramentos se não forem tratadas corretamente, o que aumenta em até 4 vezes o risco de morte dos pacientes hospitalizados. Reforçando assim, que o uso de terapias endoscópicas e de inibidores de prótons a fim de atuar como prevenção primária, são intervenções que diminuem a incidência de recidivas e consequentemente de mortalidade, proporcionando um quadro clínico mais estável ao paciente e devolvendo sua qualidade de vida (Krag, 2016).

Segundo Wannmacher (2007), a prevenção primária da HDA engloba o controle das doenças causadoras do sangramento, por meio do controle de fatores de risco modificáveis. Dessa forma, faz-se necessário por parte dos profissionais em saúde maior dedicação à educação em saúde, principalmente no âmbito da baixa complexidade e atenção primária, que proporcionam um ambiente com maior capacidade para o processo de ensino-aprendizado, estabelecimento de metas, planejamento de ações e execução de projetos educativos da população.

Uma vez que os fatores de risco que levam a hemorragia digestiva alta estão bem consolidados na literatura científica especializada, a reafirmação dos mesmos já era esperada. No entanto, ainda há bastante campo de estudo a respeito de temas que cercam a HDA, como formas de profilaxia primária e a sistematização da assistência à saúde de acordo com perfil epidemiológico de cada região. Os estudos incluídos como amostra final são oriundos de outros países que não o Brasil, podendo não representar com fidelidade perfis demográficos e epidemiológicos nacionais, trazendo a reflexão de que seriam necessários estudos recentes com evidências que reflitam o curso da doença no Brasil, e como os serviços de saúde brasileiros prestam ou sistematizam a assistência à saúde em casos de hemorragia digestiva alta. 


\section{Conclusão}

Os estudos analisados nesta revisão reafirmam o que já é evidenciado na literatura, reafirmando como principais fatores de risco para o desenvolvimento da HDA: úlcera péptica, varizes esofágicas, cirrose hepática, uso de medicamentos agressores da mucosa gástrica como os anti-inflamatórios não esteróides (AINEs), infecção por Helicobacter Pylori, sexo masculino, idade avançada, insuficiência renal e estilos de vida não saudáveis, com destaque para alcoolismo e tabagismo.

Contudo, tais fatores de risco não são de domínio público no que tange a compreensão do que causam seus desenvolvimentos, como no caso das úlceras pépticas e varizes esofágicas, ou a compreensão de quais danos para a saúde hábitos não saudáveis como a não higienização das mãos e alimentos, a adoção ou manutenção de maus hábitos alimentares, a automedicação, alcoolismo e tabagismo podem trazer.

A difusão de conhecimentos que proporcionem o entendimento da fisiopatologia de fatores de risco para HDA, como a infecção por H. pylori, o desenvolvimento e úlcera péptica e de como chegam ao ponto de um episódio hemorragia digestiva alta, além de quais os impactos que essa hemorragia trará na morbimortalidade dos afetados, podem gerar mudanças de curto e longo prazo em relação ao autocuidado, no que diz respeito a maior adesão de hábitos alimentares saudáveis e acompanhamento, através de consultas e exames, de possíveis sinais e sintomas indicativos de complicações gastrointestinais, além de uma menor adesão ou frequência de hábitos e práticas que propiciem o surgimento de fatores de risco que levem a HDA.

Ressalta-se, por fim, a necessidade da participação efetiva da atenção primária à saúde neste processo, estimulando o fortalecimento de práticas voltadas à educação popular, objetivando reduzir a grande maioria das causas que levam ao desenvolvimento de episódios da HDA. Sugere-se que estudos futuros sejam elaborados com o intuito de investigar outros fatores de risco em grupos populacionais específicos, permitindo com que seja possível estimar seus desenvolvimentos e desfechos clínicos, possibilitando com que esta temática seja melhor avaliada e trabalhada de acordo com o avanço da área.

\section{Referências}

Abd ElRahim, A. Y., Fouad, R., Khairy, M. et al (2018). Eficácia de carvedilol versus propranolol versus ligadura elástica de varizes para prevenção primária de sangramento de varizes. Hepatol Int 12, 75-82. https://doi.org/10.1007/s12072-017-9835-9

Araujo, S. M., Alexandre, A. C. S. \& Albuquerque, A. P. S. (2016). Achados endoscópicos em usuários acometidos por hemorragia digestiva alta em situação de emergência. Revista de Enfermagem UFPE Online, 10(5), 1694-1700. https://doi.org/10.5205/reuol.9003-78704-1-SM.1005201615

Benites-Goñi, H., Brañez-Condorena, A., Monge-Del Valle, F., Medina-Morales, B., Cárdenas-Vela, I., Asencios-Cusihuallpa, J., Castillo-Plasencia, C., Bardalez-Cruz, P., Dávalos-Moscol, M., \& Taype-Rondan, A. (2018). Adherencia a la guía de práctica clínica institucional sobre hemorragia digestiva alta, en un hospital de Lima - Perú. Revista de Gastroenterología del Perú, 40(2), 115-126. http://www.scielo.org.pe/scielo.php?script=sci_arttext\&pid=S1022$51292020000200115 \& \operatorname{lng}=\mathrm{es} \& \operatorname{tlng}=\mathrm{es}$

Brasil. (2014). Diretrizes Metodológicas Sistema GRADE - manual de graduação da qualidade da evidência e força de recomendação para tomada de decisão em saúde. Ministério da Saúde. Secretaria de Ciência, Tecnologia e Insumos Estratégicos Departamento de Ciência e Tecnologia. https://bvsms.saude.gov.br/bvs/publicacoes/diretrizes_metodologicas_sistema_grade.pdf

Condo, A. I. C. (2018). Perfil epidemiológico de hemorragia digestiva alta asociada a aines en el Hospital Guasmo Sur [Universidad de Guayaquil. Facultad de Ciencias Médicas]. Repositorio Universidad de Guayaquil. http://repositorio.ug.edu.ec/handle/redug/30545

Couto, G. et al. (2010). Hemorragia digestiva alta associada ao consumo de ácido acetilsalicílico e de anti-inflamatórios não esteróides em Portugal, [s. l.], v. 17, p. 7, 2010. Jornal Português de Gastrenterologia.

Giordiano--Nappi, J. et al (2008). Aspectos endoscópicos no manejo da úlcera péptica gastroduodenal, 8. https://doi.org/10.1590/S0100-69912008000200010.

Jiménez, César Eduardo, Randial, Leonardo, \& Quiroga, Fernando. (2019). Manejo endovascular de la hemorragia digestiva, experiencia del Hospital Universitario Clínica San Rafael. Revista Colombiana de Cirugía, 34(3), 234-244. https://doi.org/10.30944/20117582.436

Kim, S. H., Yun, J. M., Chang, C. B., Piao, H., Yu, S. J., \& Shin, D. W. (2016). Prevalence of upper gastrointestinal bleeding risk factors among the general population and osteoarthritis patients. World journal of gastroenterology, 22(48), 10643-10652. https://doi.org/10.3748/wjg.v22.i48.10643

Kim, J. (2017). Fatores associados a ressangramento em pacientes com sangramento de úlcera péptica: análise do estudo coreano de sangramento de úlcera péptica (K-PUB), Gut Liver, p. 7, 14 set. 2017. https://doi.org/10.5009/gnl17138.

Kiringa, S. K., Quinlan, J., Ocama, P., Mutyaba, I., \& Kagimu, M. (2020). Prevalence, short term outcome and factors associated with survival in patients suffering from upper gastrointestinal bleeding in a resource limited-setting, the case of Mulago hospital in Kampala, Uganda. African health sciences, 20(1), 426-436. https://doi.org/10.4314/ahs.v20i1.49 
Krag, M., Perner, A., Wetterslev, J. et al (2016). Profilaxia de úlcera de estresse com um inibidor da bomba de prótons versus placebo em pacientes criticamente enfermos (ensaio SUP-ICU): protocolo de estudo para um ensaio clínico randomizado. Trials 17, 205. https://doi.org/10.1186/s13063-016-1331-3

Krag, M. (2018). Pantoprazol em pacientes com risco de sangramento gastrointestinal na UTI. The New England Journal of Medicine. 10.1056 / NEJMoa1714919.

Lee, Y. J., Min, B. R., Kim, E. S., Park, K. S., Cho, K. B., Jang, B. K., Chung, W. J., Hwang, J. S., \& Jeon, S. W. (2016). Predictive factors of mortality within 30 days in patients with nonvariceal upper gastrointestinal bleeding. The Korean journal of internal medicine, 31(1), 54-64. https://doi.org/10.3904/kjim.2016.31.1.54

Martins, A. A. L., Silva, A. M. F., Andrade, F. G., Garcia, H. C. R., Brito, A. P. S. O., \& Maneschy, R. B. (2019). Hemorragia digestiva alta diagnóstico e tratamento: uma revisão de literatura. Pará Research Medical Journal, 3(07). https://doi.org/http://dx.doi.org/10.4322/prmj.2019.007

Mauro, A., De Grazia, F., Lenti, M. V., Penagini, R., Frego, R., Ardizzone, S., Savarino, E., Radaelli, F., Bosani, M., Orlando, S., Amato, A., Dinelli, M., Ferretti, F., Filippi, E., Vecchi, M., Stradella, D., Bardone, M., Pozzi, L., Rovedatti, L., Strada, E., \& Di Sabatino, A. (2021). Upper gastrointestinal bleeding in COVID-19 inpatients: Incidence and management in a multicenter experience from Northern Italy. Clinics and research in hepatology and gastroenterology, 45(3), 101521. https://doi.org/10.1016/j.clinre.2020.07.025

Melo, N. F. R. (2015). Hemorragia digestiva alta: Marcadores de gravidade para a Sala de Emergência (Publication No. 37659) [Doctoral dissertation, Instituto de Ciências Biomédicas Abel Salazar-Universidade do Porto]. Repositório Aberto da Universidade do Porto. https://repositorioaberto.up.pt/handle/10216/88197

Moledina, S. M., \& Komba, E. (2017). Risk factors for mortality among patients admitted with upper gastrointestinal bleeding at a tertiary hospital: a prospective cohort study. BMC gastroenterology, 17(1), 165. https://doi.org/10.1186/s12876-017-0712-8

Paczek, R., Espirito Santo, D., \& Galvan, C. (2020). Utilização de equipamentos de proteção individual em unidade endoscópica. Revista de Enfermagem UFPE on line, 14. https://doi.org/10.5205/1981-8963.2020.243993

Pinto, C., Parra, P., Magna, J., Gajardo, A., Berger, Z., Montenegro, C., \& Muñoz, P. (2020, March). Hemorragia digestiva alta varizes e sem varizes: mortalidad intrahospitalaria y características clínicas en un hospital universitario (2015-2017). Revista Médica de Chile, 148(3), 288-294. https://doi.org/http://dx.doi.org/10.4067/S0034-98872020000300288

Santos, C. M. d. C., Pimenta, C. A. d. M., \& Nobre, M. R. C. (2007). A estratégia PICO para a construção da pergunta de pesquisa e busca de evidências. Rev. Latino-Am. Enfermagem, 15(3), 508-511. https://doi.org/10.1590/S0104-11692007000300023.

Soares, C. B., Hoga, L. A. K., Peduzzi, M., Sangaleti, C., Yonekura, T., \& Silva, D. R. A. D. (2014). Revisão integrativa: conceitos e métodos utilizados na enfermagem. Rev. esc. enferm. USP, 48(02). https://doi.org/10.1590/S0080-6234201400002000020

Soares, D. et al (2014). Hemorragia digestiva alta, [s. 1.], p. 8, 2014. Biblioteca virtual em saúde.

Souza, A. H. d. (2017). Tecnologia operacional visando à assistência de enfermagem no setor de endoscopia (Publication No. 906467) [Escola de Enfermagem Aurora de Afonso Costa]. Biblioteca Virtual em Saúde. https://pesquisa.bvsalud.org/enfermeria/resource/pt/biblio-906467

Souza, M. T. d., Silva, M. D. d., \& Carvalho, R. d. (2010). Revisão integrativa: o que é e como fazer. Einstein, 8(1), 102-106. https://doi.org/10.1590/S167945082010RW1134

Tang, B., \& Xiao, S. (2020). Logistic regression analysis of risk factors for upper gastrointestinal bleeding induced by PCI in combination with double antiplatelet therapy for STEMI patients. Acta gastro-enterologica Belgica, 83(2), 245-248. https://pubmed.ncbi.nlm.nih.gov/32603042

Vomero, N., \& Colpo, E. (2014). Cuidados nutricionais da úlcera péptica. Arquivos Brasileiros de Cirurgia Digestiva. https://doi.org/10.1590/S010267202014000400017

Wannmacher, L. (2007). Sangramento digestivo alto: prevenção e tratamento., 4(10). https://www.paho.org/bra/dmdocuments/V4N10_SET2007_HEMORR AG_DIGEST_LAYOUT_FIM_2.pdf

Yang, S. (2017). Risco de ressangramento e mortalidade em pacientes cirróticos com sangramento de úlcera péptica: um estudo de coorte nacional de 12 anos. $P M C, 18,10.1371$ / journal.pone.0168918. 